Article

\title{
Active Fault Tolerant Control of Faulty Uncertain Neutral Time-Delay Systems
}

\author{
Benjemaa Rabeb ${ }^{1}$, Elhsoumi Aicha ${ }^{2}$, Bel Hadj Ali Saloua ${ }^{2, *}$ \\ ${ }^{1}$ Gabes University: MACS LR16ES22, National Engineering school of Gabes, 6029, Tunisia \\ 2 ElManar University, Preparatory Institute for Engineering studies-EIManar, 1068, Tunisia \\ ${ }^{*}$ Correspondence: Saloua.belhadjali@enit.rnu.tn;
}

How to cite this paper: Rabeb, B., Aicha, E., \& Saloua, B. H. A. (2021). Active fault tolerant control of faulty uncertain neutral time-delay systems. Journal of Artificial Intelligence and Big Data, 1(1), 18-30. Retrieved from https://www.scipublications.com/journal/index.php/jaibd/article/view/59

Received: July 2, 2021

Accepted: September 3, 2021

Published: September 4, 2021

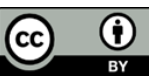

Copyright: (c) 2021 by the authors. Submitted for possible open access publication under the terms and conditions of the Creative Commons Attribution (CC BY) license (http://creativecommons.org/licenses /by/4.0/).

\begin{abstract}
The present paper attempts to investigate the problem of Fault Tolerant Control for a class of uncertain neutral time delay systems. In the first time, we consider an additive control that is based on adding a term to the nominal law when the fault occurs. This approach will be designed in three steps. The first step is fault detection while the second one is fault estimation. For these two steps, we consider the adaptive observer to guarantee the detection and estimation of the fault. The third step is the fault compensation. Lyapunov method and Linear Matrix Inequality (LMI) techniques were considered to improve the main method. Second, we propose a Pseudo Inverse Method "PIM" and determine the error between the closed loop and the nominal system. Finally, simulation results are presented to prove the theoretical development for an example of an uncertain neutral time delay system.
\end{abstract}

Keywords: Uncertain neutral time delay system, Active Fault Tolerant Control (AFTC), LMIs, additive control, PIM.

\section{Introduction}

In recent years, there has been a burst of research activities in the area of time-delay control systems [1-3].

Time delay systems can be classified into two different categories: the first one expressed by retarded functional differential equations (RFDE) and the second one described by neutral functional differential equations (NFDE).

The presence of delays more precisely for neutral time-delay system induces poor performance and instability in closed-loop systems. Furthermore, some faults can be occur during the system operation.

Fault is understood as any sort of malfunction that occurs in actuator, sensor or process level and that leads to any deviation from the nominal condition.

The system treated in this work has the particularity of the uncertain elements.

Uncertain RFDE systems have been studied in $[1,3,4,9,10]$. Their objective was to determine the stability and control of this type of system.

Uncertain NFDE systems have been studied in $[8,11]$ to achieve the adaptive robust control.

Benjemaa, R.; and al. in [1] studied the stability and active fault tolerant control for a class of certain neutral time delay systems; this work is a continuity of [1] such that, we will consider the neutral time delay systems with parametric uncertainty and we will compare two methods of active fault tolerant control.

The presence of parametric uncertainty, which can cause important error in system parameters identification, guide to more complexity of the system model. Thus, neutral variable time-delay and parameter uncertainty are sources of the oscillation generation, instability and poor performance. 
An adaptive robust control scheme was proposed in [7] to stabilize uncertain neutral time-delay systems, as well as in [8]. The authors proposed an adaptive Robust control of uncertain neutral time-delay systems.

However, the main drawback of this work is that it assumes the delayed state of the system in the adaptive process.

In this article, the uncertain neutral time-delay systems are studied, and a new active fault-tolerant control is proposed to this type of system.

The comparison between additive and PIM control for uncertain neutral time delay systems is the principal contribution of this paper.

First, the additive control approach (AFTC) will be presented. The main idea of this control is based on adding a term to the nominal control law when the fault occurs, to compensate the fault effect.

Second, the Pseudo-Inverse Method (PIM) will be designed for this type of system based on the error determination between the closed loop system and the nominal one.

The remainder of the paper is organized as follows. The problems statement is given in section 2. In section 3, the main results of the AFTC are presented; the method of additive control (fault detection, estimation and fault compensation) and the PIM, for uncertain neutral time-delay systems.

Simulation results are presented in section 4 to demonstrate the efficacy of the proposed method and finally, some concluding remarks are drawn in section 5 .

\section{Notation:}

Let us consider the following notations used in this paper.

$R^{n}$ is the n-dimensional Euclidean space $R^{n \times m}$ is the space of all real matrices, and the superscript "T" denotes the transpose and LMI denote Linear Matrix Inequality. I is the identity matrix of appropriate dimension. "*" denote the transposed elements in the symmetric position.

\section{Problems Statement}

Consider a class of linear uncertain neutral time delay systems described by the following state space equation:

$$
\left\{\begin{array}{c}
\dot{\xi}(t)=\left(A_{0}+\Delta A_{0}\right) \xi(t)+\left(A_{1}+\Delta A_{1}\right) \xi(t-h)+ \\
\left(A_{2}+\Delta A_{2}\right) \dot{\xi}(t-d)+(B+\Delta B) u(t) \\
y(t)=C \xi(t) \\
\xi(t)=\varphi(t) \quad ; \quad t \in\left[\begin{array}{lll}
-\tau, & 0
\end{array}\right]
\end{array}\right.
$$

Where $\xi(t) \in R^{n}$ is the state vector, $u(t) \in R^{m}$ is the input vector, $y(t) \in R^{p}$ is the output vector. $h>0$ and $d>0$ are respectively state and its derivative delay.

$\mathrm{A}_{0}, \mathrm{~A}_{1}, \mathrm{~A}_{2}, \mathrm{~B}$ and $\mathrm{C}$ are constant matrices with appropriate dimensions. $\Delta A_{0}, \Delta A_{1}$, $\Delta A_{2}$ and $\Delta B$ are matrix-valued functions representing the parameter uncertainties in the system model; and $\varphi(t)$ is a given continuous vector valued initial function.

The parameter uncertainties considered here are assumed that:

$$
\begin{aligned}
& \Delta A_{0}=J_{1} \delta W_{1} \\
& \Delta A_{1}=J_{2} \delta W_{2} \\
& \Delta A_{2}=J_{3} \delta W_{3} \\
& \Delta B=J_{4} \delta W_{4}
\end{aligned}
$$

Where: $J_{1}, J_{2}, J_{3}, J_{4}, W_{1}, W_{2}, W_{3}, W_{4}$ and $\delta$ are constant matrices with appropriate dimensions. 
Consider the uncertain neutral delay system affected by actuator fault:

$$
\left\{\begin{array}{c}
\dot{\xi}(t)=\left(A_{0}+\Delta A_{0}\right) \xi(t)+\left(A_{1}+\Delta A_{1}\right) \xi(t-h)+ \\
\left(A_{2}+\Delta A_{2}\right) \dot{\xi}(t-d)+(B+\Delta B) u(t)+F f(t) \\
y(t)=C \xi(t) \\
\xi(0)=\xi_{0} \quad ; \quad t \leq 0
\end{array}\right.
$$

Where $\mathrm{F}$ is a constant matrix with appropriate dimensions and $\mathrm{f}(\mathrm{t})$ is actuator fault.

The presence of fault causes system damage. However, two fault tolerant control (FTC) approaches are proposed for uncertain neutral time delay systems possessing the ability to maintain nominal targets despite the presence of a fault.

\section{Main results}

In this section, the two methods, as detailed in introduction are considered to design the FTC for the system (2).

\subsection{Additive Control}

\subsubsection{Fault detection and estimation}

Considering the system (2). As a first step, the following adaptive observer is used to detect the actuator fault:

$$
\left\{\begin{array}{c}
\dot{\xi}_{m}(t)=A_{0} \xi_{m}(t)+A_{1} \xi_{m}(t-h)+A_{2} \dot{\xi}_{m}(t-d)+ \\
B u(t)+L\left(y(t)-y_{m}(t)\right)+H\left(y(t-h)-y_{m}(t-h)\right) \\
y_{m}(t)=C \xi_{m}(t) \\
\xi(0)=\xi_{0} ; t \leq 0 \\
r(t)=V\left(y(t)-y_{m}(t)\right)
\end{array}\right.
$$

Where $\xi_{m}(t) \in R^{n}$ and $y_{m}(t) \in R^{p}$ denote respectively, the state and the output vector of the observer.

$\mathrm{V}$ : The residue weight.

The model error and residual are defined as follows:

$$
\left\{\begin{array}{c}
\dot{e}(t)=\left(A_{0}-L C\right) e(t)+A_{2} \dot{e}(t-d)+ \\
\left(A_{1}-H C\right) e(t-h)+F f(t)+\Delta A_{0} \xi(t)+ \\
\Delta A_{1} \xi(t-h)+\Delta A_{2} \dot{\xi}(t-d)+\Delta B u(t) \\
r(t)=V e(t) \\
e(0)=e_{0} ; t \leq 0
\end{array}\right.
$$

To calculate $\mathrm{L}$ and $\mathrm{H}$; we consider the following theorem:

Theorem 1.[1] 
Given scalar $\mathrm{h}>0$, considering the following Linear Matrix Inequality such that, if there exist symmetric positive definite matrices $\mathrm{P}, \mathrm{Q}, \mathrm{W}, Z_{1}, Z_{2}, Z_{3}, \mathrm{G} \in R^{n^{*} n}$, and $Y_{1}, Y_{2} \in R^{n^{*} p}, M_{i}, \quad N_{i} \in R^{n^{*} n}, \quad \mathrm{i}=1, . ., 4$, such that:

$\left[\begin{array}{cccccccccc}\phi_{11} & \phi_{12} & \phi_{13} & \phi_{14} & \phi_{15} & h M_{1} & h N_{1} & \phi_{18} & \phi_{19} & \phi_{110} \\ * & \phi_{22} & \phi_{23} & \phi_{24} & \phi_{25} & h M_{2} & h N_{2} & \phi_{28} & \phi_{29} & \phi_{210} \\ * & * & \phi_{33} & A_{2}^{T} N_{4}^{T} & 0 & h M_{3} & h N_{3} & 0 & 0 & 0 \\ * & * & * & -Z_{1} & -A_{2}^{T} P F & h M_{4} & h N_{4} & A_{2}^{T} P & h A_{2}^{T} P & 0 \\ * & * & * & * & \phi_{55} & 0 & 0 & F^{T} P & h F^{T} P & h F^{T} P \\ * & * & * & * & * & -h Z_{2} & 0 & 0 & 0 & 0 \\ * & * & * & * & * & * & -h Z_{3} & 0 & 0 & 0 \\ * & * & * & * & * & * & * & -P Z_{1}^{-1} P & 0 & 0 \\ * & * & * & * & * & * & * & * & -h P Z_{2}^{-1} P & 0 \\ * & * & * & * & * & * & * & * & * & -h P Z_{3}^{-1} P\end{array}\right] \prec 0$

Where:

$$
\begin{aligned}
& \phi_{11}=P A_{0}+A_{0}^{T} P-Y_{1} C-C^{T} Y_{1}^{T}+Q+M_{1}+M_{1}^{T}+N_{1}+N_{1}^{T} \\
& \phi_{12}=P A_{1}-Y_{2} C-A_{0}^{T} P A_{2}+C^{T} Y_{1}^{T} A_{2}-M_{1}+M_{2}^{T}-N_{1}\left(A_{2}+I\right)+N_{2}^{T} \\
& \phi_{13}=M_{3}^{T}+N_{1} A_{2}+N_{3}^{T} \\
& \phi_{14}=M_{4}^{T}+N_{4}^{T} \\
& \phi_{15}=-A_{0}^{T} P F+C^{T} Y_{1}^{T} F \\
& \phi_{18}=A_{0}^{T} P-C^{T} Y_{1}^{T} \\
& \phi_{19}=\phi_{110}=h A_{0}^{T} P-h C^{T} Y_{1}^{T} \\
& \phi_{22}=-A_{2}^{T} P A_{1}-A_{1}^{T} P A_{2}+A_{2}^{T} Y_{2} C+C^{T} Y_{2}^{T} A_{2}-Q+W-M_{2}-M_{2}^{T}-N_{2}\left(A_{2}+I\right) \\
& -\left(A_{2}+I\right)^{T} N_{2}^{T} \\
& \phi_{23}=-M_{3}^{T}+N_{2} A_{2}-\left(A_{2}+I\right)^{T} N_{3}^{T} \\
& \phi_{24}=-M_{4}^{T}-\left(A_{2}+I\right)^{T} N_{4}^{T} \\
& \phi_{25}=-A_{2}^{T} P F-A_{1}^{T} P F+C^{T} Y_{2}^{T} F \\
& \phi_{28}=A_{1}^{T} P-C^{T} Y_{2}^{T} \\
& \phi_{29}=\phi_{210}=h A_{2}^{T} P-h C^{T} Y_{2}^{T} \\
& \phi_{33}=-W+N_{3} A_{2}+A_{2}^{T} N_{3}^{T} \\
& \phi_{55}=-2 F^{T} P F+G
\end{aligned}
$$

Then:

$$
\begin{aligned}
& Y_{1}=P L \\
& Y_{2}=P H
\end{aligned}
$$

In step 2, we use the same observer to estimate the fault with the following form: 


$$
\left\{\begin{array}{l}
\dot{\hat{\xi}}(t)=A_{0} \hat{\xi}(t)+A_{1} \hat{\xi}(t-h)+A_{2} \dot{\hat{\xi}}(t-d)+B u(t)+F \hat{f}(t) \\
+L[y(t)-\hat{y}(t)]+H[y(t-h)-\hat{y}(t-h)] \\
\quad \hat{y}(t)=C \hat{\xi}(t) \\
r(t)=V[y(t)-\hat{y}(t)] \\
\hat{\xi}(0)=\hat{\xi}_{0} ; \quad t \leq 0
\end{array}\right.
$$

Where:

$$
\dot{\hat{f}}(t)=-\Gamma F r(t)
$$

To find $\Gamma$ the following theorem is considered:

Theorem 2. [2]

If there exists $\mathrm{R}>0, \mathrm{~S}>0$ and $\mathrm{T}>0$ symmetric positive matrices such that:

$$
\left[\begin{array}{ccccc}
2 R A_{0}-2 S C+C^{T} Z C+T & R A_{2} & -R F+C^{T} V^{T} F & 0 & 0 \\
* & -(1-\beta) T & 0 & 0 & 0 \\
* & * & \Gamma^{2} & 0 & 0 \\
* & * & * & 0 & 0 \\
* & * & * & * & 0
\end{array}\right] \prec 0
$$

With:

$$
\begin{gathered}
S=H R \\
Z=V^{T} V
\end{gathered}
$$

\subsubsection{Fault compensation}

To ensure a zero-static error between the system output and the desired one, we add an integrator to the control law. Therefore, we can design the following state feedback controller to stabilize the closed-loop system:

$$
u(t)=K \xi(t)+\varepsilon(t)
$$

Where:

$$
\varepsilon(t)=\int_{0}^{t}\left(y_{r}(\theta)-y(\theta)\right) d \theta
$$

And

$$
\dot{\varepsilon}(t)=y_{r}(t)-y(t)
$$

Where:

$\varepsilon$ : The static error.

$y_{r}$ : The desired output.

Where $\mathrm{K}$ the feedback matrix gain obtained by LMI resolution in the following theorem. 


\section{Theorem 3.}

For given scalars $\tau^{*} \succ 0$ and $\mu \prec 1$, the system (2) with the matrices $A_{0}, A_{1}$, and $A_{2}$ are constant, is asymptotically stabilizable by state feedback, if there exists $P_{1}=P_{1}^{T} \succ 0, Q_{1}=Q_{1}^{T} \succ 0, Z=Z^{T} \succ 0$ and matrices with appropriate dimensions $G_{i}(\mathrm{i}=1,2,3), \mathrm{D}$ and $\mathrm{O}$ such that satisfy the following LMI:

$$
\mathrm{E}^{*}=\left[\begin{array}{cccc}
\mathrm{E}_{11}^{*} & \mathrm{E}_{12}^{*} & \mathrm{E}_{13}^{*} & \tau^{*} G_{1} \\
* & \mathrm{E}_{22}^{*} & \mathrm{E}_{23}^{*} & \tau^{*} G_{2} \\
* & * & \mathrm{E}_{33}^{*} & \tau^{*} G_{3} \\
* & * & * & -\tau^{*} Z
\end{array}\right] \prec 0
$$

Where:

$$
\begin{gathered}
\mathrm{E}_{11}^{*}=Q_{1}+G_{1}+G_{1}^{T}-A_{0} O^{T}-O A^{T}-B D-D^{T} B \\
\mathrm{E}_{12}^{*}=G_{2}^{T}-G_{1}-A_{0} O^{T}-O A_{1}^{T}-B D \\
\mathrm{E}_{13}^{*}=P_{1}+G_{3}^{T}+O-A_{0} O^{T} \\
\mathrm{E}_{22}^{*}=-(1-\mu) Q-G_{2}-G_{2}^{T}-O A_{1}^{T}-A_{1} O^{T} \\
\mathrm{E}_{23}^{*}=-G_{3}^{T}+O-A_{1} O^{T} \\
\mathrm{E}_{33}^{*}=\tau^{*} Z+O+O^{T}
\end{gathered}
$$

Then

$$
K=D O^{-T}
$$

\section{Proof theorem 3.}

Lemma 1. the system (1) is asymptotically stable if it exists $P_{1}=P_{1}^{T} \succ 0, Q_{1}=Q_{1}^{T} \succ 0$, $Z=Z^{T} \succ 0$ and the matrices of appropriate dimensions $G_{i}$ and $O_{i} \quad(\mathrm{i}=1,2,3)$ such that the following LMI is satisfied:

$$
\mathrm{E}=\left[\begin{array}{cccc}
\mathrm{E}_{11} & \mathrm{E}_{12} & \mathrm{E}_{13} & \tau^{*} G_{1} \\
* & \mathrm{E}_{22} & \mathrm{E}_{23} & \tau^{*} G_{2} \\
* & * & \mathrm{E}_{33} & \tau^{*} G_{3} \\
* & * & * & -\tau^{*} Z
\end{array}\right] \prec 0
$$

Where:

$$
\begin{gathered}
\mathrm{E}_{11}=Q_{1}+G_{1}+G_{1}^{T}-A_{0} O_{1}^{T}-O_{1} A_{0}^{T} \\
\mathrm{E}_{12}=G_{2}^{T}-G_{1}-A_{0} O_{2}^{T}-O_{1} A_{1}^{T} \\
\mathrm{E}_{13}=P_{1}+G_{3}^{T}+O_{1}-A_{0} O_{3}^{T} \\
\mathrm{E}_{22}=-(1-\mu) Q_{1}-G_{2}-G_{2}^{T}-O_{2} A_{1}^{T}-A_{1} O_{2}^{T} \\
\mathrm{E}_{23}=-G_{3}^{T}+O_{2}-A_{1} O_{3}^{T} \\
\mathrm{E}_{33}=\tau^{*} Z+O_{3}+O_{3}^{T}
\end{gathered}
$$

To prove LMI (13), we choose the following Lyapunov function: 


$$
\begin{gathered}
V(\xi, t)=\xi^{T}(t) P_{1} \xi(t)+\int_{t-h}^{t} \xi^{T}(s) Q_{1} \xi(s) d s+ \\
\int_{t-d}^{t} \dot{\xi}^{T}(s) Z \dot{\xi}(s) d s+\int_{-d}^{0}\left(\int_{t+\beta}^{t} \dot{\xi}^{T}(s) D \dot{\xi}(s) d s\right) d \beta
\end{gathered}
$$

Where the matrices $P_{1}, Q_{1}, \mathrm{Z}$ and $\mathrm{D}$ are to be determinate.

Then, the calculation of the derivative of $V(\xi, t)$ gives:

$$
\begin{gathered}
\dot{V}(\xi, t)=2 \dot{\xi}^{T}(t) P_{1} \xi(t)+\xi^{T}(t) Q_{1} \xi(t)-\xi^{T}(t-h) Q_{1} \xi(t-h) \\
+\dot{\xi}^{T}(t) Z \xi(t)-\dot{\xi}^{T}(t-d) Z \dot{\xi}(t-d) \\
+\int_{-d}^{0}\left[\dot{\xi}^{T}(t) D \dot{\xi}(t)-\dot{\xi}^{T}(t+\beta) D \dot{\xi}(t+\beta)\right] d \beta \\
=2 \dot{\xi}^{T}(t) P_{1} \xi(t)+\xi^{T}(t) Q_{1} \xi(t)-\xi^{T}(t-h) Q_{1} \xi(t-h) \\
+(1+d) \dot{\xi}^{T}(t) D \dot{\xi}(t)-\dot{\xi}^{T}(t-d) Z \dot{\xi}(t-d) \\
-\int_{-d}^{0} \dot{\xi}^{T}(t+\beta) D \dot{\xi}(t+\beta) d \beta \\
\leq 2 \dot{\xi}^{T}(t) P_{1} \xi(t)+\xi^{T}(t) Q_{1} \xi(t)-\xi^{T}(t-h) Q_{1} \xi(t-h) \\
-(1-\mu) \dot{\xi}^{T}(t) D \dot{\xi}(t)-\dot{\xi}^{T}(t-d) Z \dot{\xi}(t-d) \\
-\int_{-d}^{0} \dot{\xi}^{T}(t+\beta) D \dot{\xi}(t+\beta) d \beta
\end{gathered}
$$

The Leibniz-Newton formula provides:

$$
\xi(t)-\xi(t-h)-\int_{-d}^{0} \xi(t-s) d s=0
$$

Therefore, for matrices of appropriate dimensions $G_{i}(i=1,2,3)$ the following expression is true:

$2\left[\xi^{T}(t) G_{1}+\xi^{T}(t-h) G_{2}+\dot{\xi}^{T}(t) G_{3}\right] *\left[\xi(t)-\xi(t-h)-\int_{-d}^{0} \dot{\xi}(t-s) d s\right]=0$

Moreover, from (1) for matrices of appropriate dimensions, $O_{i}(i=1,2,3)$ we have:

$$
\begin{gathered}
2\left[\xi^{T}(t) O_{1}+\xi^{T}(t-h) O_{2}+\dot{\xi}^{T}(t) O_{3}\right] * \\
{\left[\left(A_{2}+\Delta A_{2}\right) \dot{\xi}(t-d)-\left(A_{1}+\Delta A_{1}\right) \xi(t-h)-\left(A_{0}+\Delta A_{0}\right) \xi(t)\right]=0}
\end{gathered}
$$


For a positive semi-definite matrix

$$
\Theta=\left[\begin{array}{lll}
\Theta_{11} & \Theta_{12} & \Theta_{13} \\
\Theta_{21} & \Theta_{22} & \Theta_{23} \\
\Theta_{31} & \Theta_{32} & \Theta_{33}
\end{array}\right] \geq 0
$$

The following expression is verified:

$$
\tau^{*} \eta^{T}(t) \Theta \eta(t)-\int_{t-d}^{t} \eta^{T}(t) \Theta \eta(t) d s \geq 0
$$

Where:

$$
\eta(t)=\left[\begin{array}{lll}
\xi^{T}(t) & \xi^{T}(t-h) & \dot{\xi}^{T}(t-d)
\end{array}\right]^{T}
$$

Then, by adding the null terms (16) and (17) to the left part of the inequality (15) and by increasing $\dot{V}(\xi, t)$ in the same inequality by the term (18), this allows us to express $\dot{V}(\xi, t)$ as follows:

$$
\dot{V}(\xi, t) \leq \eta^{T}(t) \Delta \eta(t)-\int_{t-d}^{t} \Omega^{T}(t, s) \psi \Omega(t, s) d s
$$

Where:

$$
\begin{gathered}
\Omega(t, s)=\left[\begin{array}{lll}
\eta^{T}(t) & \dot{\xi}^{T}(s)
\end{array}\right]^{T} \\
\Delta=\left[\begin{array}{lll}
\mathrm{E}_{11}+\tau^{*} \Theta_{11} & \mathrm{E}_{12}+\tau^{*} \Theta_{12} & \mathrm{E}_{13}+\tau^{*} \Theta_{13} \\
\mathrm{E}_{21}+\tau^{*} \Theta_{21} & \mathrm{E}_{22}+\tau^{*} \Theta_{22} & \mathrm{E}_{23}+\tau^{*} \Theta_{23} \\
\mathrm{E}_{31}+\tau^{*} \Theta_{31} & \mathrm{E}_{32}+\tau^{*} \Theta_{32} & \mathrm{E}_{33}+\tau^{*} \Theta_{33}
\end{array}\right] \\
\psi=\left[\begin{array}{llll}
\Theta_{11} & \Theta_{12} & \Theta_{13} & G_{1} \\
\Theta_{21} & \Theta_{22} & \Theta_{23} & G_{2} \\
\Theta_{31} & \Theta_{32} & \Theta_{33} & G_{3} \\
G_{1}^{T} & G_{2}^{T} & G_{3}^{T} & D
\end{array}\right]
\end{gathered}
$$

If $\Delta \prec 0$ and $\psi \succ 0$, then, there exists $\varepsilon$ sufficiently small such that $\dot{V}(\xi, t) \prec-\varepsilon\|\xi(t)\|^{2}$, we ensure then the asymptotically stability of the system (1). More precisely, if we select $D \succ 0$, the matrix $\Theta$ can be chosen as:

$$
\Theta=\left[\begin{array}{l}
\Theta_{1} \\
\Theta_{2} \\
\Theta_{3}
\end{array}\right]=\left[\begin{array}{l}
G_{1} \\
G_{2} \\
G_{3}
\end{array}\right] D^{-1}\left[\begin{array}{l}
G_{1} \\
G_{2} \\
G_{3}
\end{array}\right]^{T}
$$

Which allows having

$$
\Theta \geq 0 \text { and } \quad \psi \geq 0
$$

In this case $\Delta \prec 0$ is equivalent to $\mathrm{E} \prec 0$ according to Schur complement. For theorem 3 based on Lemma 1, we will determine the return gain $\mathrm{K}$. 
Consider the following hypothesis $\theta_{3}=\theta_{2}=\theta_{1}$ and substitute the control law in (19), the closed loop system then becomes:

$$
\left\{\begin{array}{c}
\dot{\xi}(t)=\left(\bar{A}_{0}+\bar{B} K\right) \xi(t)+\bar{A}_{1} \xi(t-h)+\bar{A}_{2} \dot{\xi}(t-d)+F f(t) \\
\xi(t)=\varphi(t) ; \quad t \in[-\tau, 0]
\end{array}\right.
$$

To determine the return gain $\mathrm{K}$, it suffices to replace the matrix $\bar{A}_{0}$ by $\left(\bar{A}_{0}+\bar{B} K\right)$ in LMI (13) of Lemma 1.

Where $\bar{A}_{0}=A_{0}+\Delta A_{0}, \bar{A}_{1}=A_{1}+\Delta A_{1}, \bar{A}_{2}=A_{2}+\Delta A_{2}$ and $\bar{B}=B+\Delta B$.

By developing the computation, we end up with a nonlinear LMI. To solve this problem, we recourse to the dual system.

A system is stable. Then, its dual is also stable, since solution of:

$$
\operatorname{det}\left(s I-\left(\bar{A}_{0}+\bar{B} K\right)-\bar{A}_{1} e^{-\tau^{*} s}-\bar{A}_{2} e^{-\tau^{*} s}\right)=0
$$

Is the same that of:

$$
\operatorname{det}\left(s I-\left(\bar{A}_{0}+\bar{B} K\right)^{T}-\bar{A}_{1}^{T} e^{-\tau^{*} s}-\bar{A}_{2}^{T} e^{-\tau^{*} s}\right)=0
$$

Therefore, is equivalent to the following system:

$$
\left\{\begin{array}{c}
\dot{\xi}(t)=\left(\bar{A}_{0}+\bar{B} K\right)^{T} \xi(t)+\bar{A}_{1}^{T} \xi(t-h)+\bar{A}_{2}^{T} \dot{\xi}(t-d) \\
\xi(t)=\varphi(t) ; \quad t \in[-\tau, 0]
\end{array}\right.
$$

To determine the return gain we replace $\bar{A}_{0}$ by $\left(\bar{A}_{0}+\bar{B} K\right)^{T}$ in (13) and we obtain (12). We propose computing a new control law $u_{a d}(t)$ to be added to the nominal one to compensate the fault effect on the system. With $u_{a d}(t)$ satisfies the following equation:

$$
\bar{B} u_{a d}(t)+F f(t)=0
$$

Then:

$$
u_{a d}(t)=-\bar{B}^{+} F f(t)
$$

Where:

$$
\bar{B}=B+\Delta B
$$

In addition, $\bar{B}^{+}$is the pseudo-inverse of matrix $\bar{B}$.

\subsection{PIM for uncertain neutral time-delay system}

In this part, the Pseudo-Inverse-Method is based on the error determination between the closed loop system and the nominal one. 
The main idea of this method is to modify the constant feedback gains of nominal system, then, synthesized controller gains that can stabilize the faulty systems.

The reference model is defined as:

$$
\dot{\xi}(t)=X_{1} \xi(t)+X_{2} \xi(t-h)+X_{3} \dot{\xi}(t-d)
$$

Where $X_{1}, X_{2}$ and $X_{3}$ are given.

Considering the state feedback, the set of admissible controls is:

$$
u_{\text {nom }}(t)=G_{\text {nom }} v_{\text {ref }}(t)-K_{\text {nom }} \xi(t)
$$

Where:

$v_{\text {ref }}(t)$ is the reference signal.

$G_{\text {nom }}$ and $K_{\text {nom }}$ are the nominal feedback matrix obtained as:

$$
\begin{gathered}
K_{\text {nom }}=(B+\Delta B)^{+}\left[\left(A_{0}+\Delta A_{0}\right)+\left(A_{1}+\Delta A_{1}\right)+\left(A_{2}+\Delta A_{2}\right)\right. \\
\left.-X_{1}-X_{2}-X_{3}\right] \\
G_{\text {nom }}=\left[C \left(-\left(A_{0}+\Delta A_{0}\right)-\left(A_{1}+\Delta A_{1}\right)-\left(A_{2}+\Delta A_{2}\right)\right.\right. \\
\left.\left.+(B+\Delta B) K_{\text {nom }}\right)^{-1}(B+\Delta B)\right]^{-1}
\end{gathered}
$$

When the fault is detected, the system (1) can be presented as:

$$
\left\{\begin{array}{c}
\dot{\xi}(t)=\left(A_{0 f}+\Delta A_{0 f}\right) \xi(t)+\left(A_{1 f}+\Delta A_{1 f}\right) \xi(t-h) \\
+\left(A_{2 f}+\Delta A_{2 f}\right) \dot{\xi}(t-d)+\left(B_{f}+\Delta B_{f}\right) u_{P I M}(t) \\
y(t)=C_{f} \xi(t) \\
\xi(0)=0, \quad \forall \quad t \in[-\tau, 0]
\end{array}\right.
$$

Where $u_{P I M}(t)$ represent the control law of the PIM and it is represented as:

$$
u_{P I M}(t)=-K_{f} \xi(t)+G_{f} v_{r e f}(t)
$$

Where $K_{f}$ and $G_{f}$ are the new feedback gain matrices to be determined. The expressions of the controllers gain can be represented as follows: 


$$
\begin{gathered}
K_{f}=\arg \min \|\left(\left(A_{0}+\Delta A_{0}\right)+\left(A_{1}+\Delta A_{1}\right)+\left(A_{2}+\Delta A_{2}\right)\right. \\
\left.-(B+\Delta B) K_{n o m}\right) \|_{F} \\
=\left(B_{f}+\Delta B_{f}\right)^{+}\left(\left(A_{0 f}+\Delta A_{0 f}\right)+\left(A_{1 f}+\Delta A_{1 f}\right)+\left(A_{2 f}+\Delta A_{2 f}\right)\right. \\
\left.-X_{0}-X_{1}-X_{2}\right) \\
G_{f}=\arg \min \left\|(B+\Delta B) G_{n o m}-\left(B_{f}+\Delta B_{f}\right) G_{f}\right\|_{F} \\
=(B+\Delta B)\left(B_{f}+\Delta B_{f}\right) G_{n o m} \\
=\left[C _ { f } \left(-\left(A_{0 f}+\Delta A_{0 f}\right)-\left(A_{1 f}+\Delta A_{1 f}\right)-\left(A_{2 f}+\Delta A_{2 f}\right)\right.\right. \\
\left.\left.+\left(B_{f}+\Delta B_{f}\right) K_{f}\right)^{-1}\left(B_{f}+\Delta B_{f}\right)\right]^{-1}
\end{gathered}
$$

\section{Simulation Results}

In this section, a numerical example is presented to demonstrate the effectiveness of the proposed theoretical development. Considering the linear neutral time-delay system (2), with the system matrices described by:

$$
\begin{gathered}
A_{0}=\left[\begin{array}{cc}
-0.7 & 0.2 \\
0.3 & 0.1
\end{array}\right] ; \quad A_{1}=\left[\begin{array}{cc}
0.2 & 0 \\
0.1 & -0.1
\end{array}\right] ; \quad A_{2}=\left[\begin{array}{cc}
0 & -0.1 \\
0.3 & 0.2
\end{array}\right] \\
B=\left[\begin{array}{cc}
1 & 0 \\
0 & 1
\end{array}\right] ; \quad C=\left[\begin{array}{cc}
1 & 0 \\
0 & 1
\end{array}\right] ; \quad E_{1}=\left[\begin{array}{l}
0.1 \\
0.1
\end{array}\right] ; \quad E_{2}=\left[\begin{array}{c}
0.1 \\
-0.1
\end{array}\right] \\
E_{3}=\left[\begin{array}{c}
-0.1 \\
0.1
\end{array}\right] ; \quad E_{4}=\left[\begin{array}{c}
-0.1 \\
0
\end{array}\right] ; \quad E_{5}=\left[\begin{array}{c}
0 \\
-0.1
\end{array}\right] ; \quad F=\left[\begin{array}{l}
1 \\
1
\end{array}\right] \\
F_{1}=\left[\begin{array}{ll}
0.01 & 0.3
\end{array}\right] ; \quad F_{2}=\left[\begin{array}{ll}
0.01 & 0.2
\end{array}\right] ; \quad F_{3}=\left[\begin{array}{ll}
0.01 & 0.2
\end{array}\right] \\
F_{4}=F_{5}=0.02 ; \quad L=0.2 ; \quad h=d=1 \\
f(t)= \begin{cases}2 & ; 25 \leq t \leq 40 \\
0 & ; \text { otherwise }\end{cases} \\
\tau(t)=0.5 \sin (10 t)+0.5
\end{gathered}
$$

From theorem 1 and 2, we obtain respectively:

$$
L=\left[\begin{array}{cc}
16.0459 & -14.8568 \\
-9.0311 & 11.1109
\end{array}\right] \quad ; \quad H=\left[\begin{array}{cc}
13.3768 & -10.9836 \\
-7.4135 & 8.3279
\end{array}\right]
$$

And

$$
\Gamma=2.684
$$

From theorem 3, the feedback matrix gain is obtained as:

$$
K=\left[\begin{array}{ll}
-0.5406 & -0.1999 \\
-0.3194 & -1.2802
\end{array}\right]
$$




$$
K_{f}=\left[\begin{array}{cc}
2.815 & 2.2157 \\
1.6215 & 2.1157
\end{array}\right] ; \quad G_{f}=\left[\begin{array}{ll}
2 & 2 \\
2 & 2
\end{array}\right]
$$

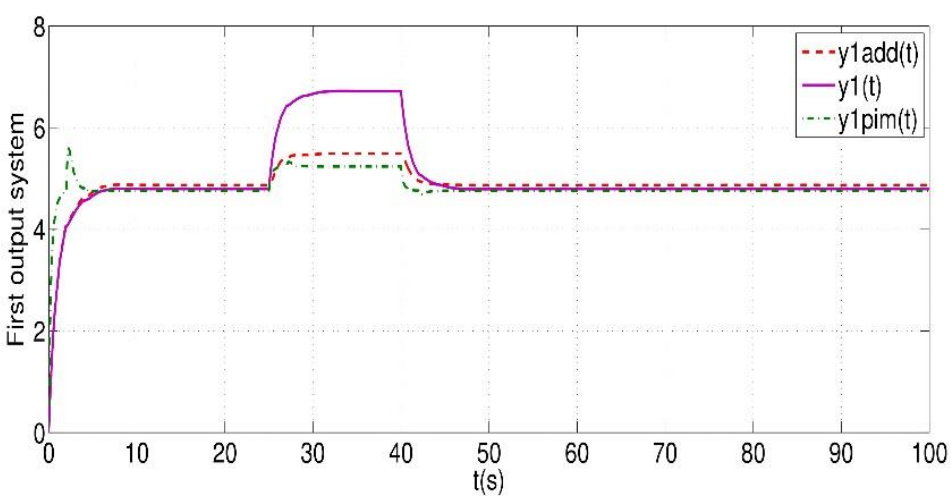

Figure 1. y1 ( $\mathrm{t}$ ) with PIM and additive control law.

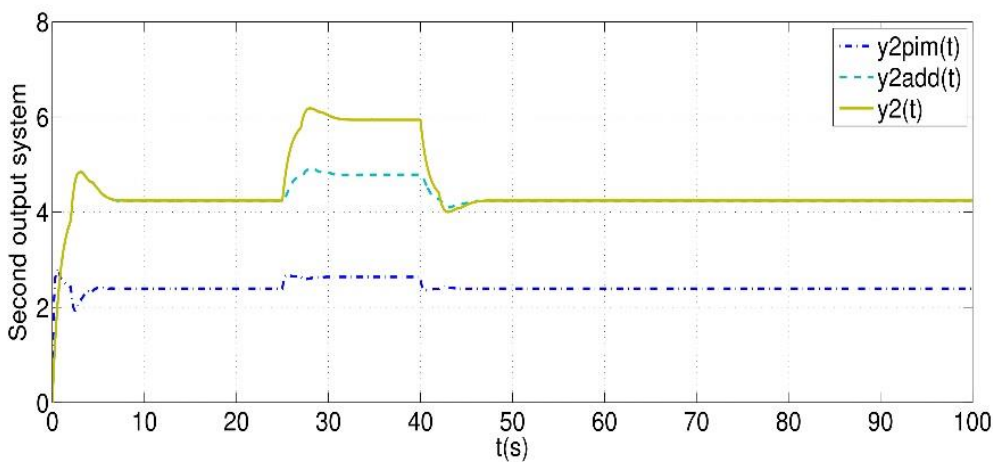

Figure 2. y2 (t) with PIM and additive control law.

By the use of additive control law method. Figure 1 and Figure 2 show that this control is able to compensate the fault (for yladd ( $\mathrm{t}$ ) and y2add (t)).

The PIM method can minimize the error between the faulty system and the reference one, Figure 1 (y1PIM (t)), but it is not the case in Figure 2 (y2PIM (t)) where the performance is minimized.

\section{Conclusion}

In this paper, two methods of active fault tolerant control are presented for an uncertain neutral time delay system.

With an additive control law added to the nominal control, the proposed AFTC achieves the minimization of fault effects.

By using a Pseudo-Inverse Method (PIM), the performance satisfaction is guaranteed for y1PIM ( $t$ ) but for y2PIM ( $t$ ) is not totally guaranteed. A numerical example has been given to show the effectiveness and limitation of the proposed methods.

\section{References}

[1] Benjemaa, R.; Elhsoumi A.; and Bel Hadj Ali, S."Stability and Active Fault Tolerant Control design for a class of neutral time delay systems". In: International Conference on Signal, Control and Communication (SCC) 2019. IEEE, 2019. pp. 24-29.

[2] Benjemaa, R.; Elhsoumi, A.; and Bel Hadj Ali, S. "Active Fault Tolerant Control for uncertain neutral time delay system". In the International Multi-Conference on Systems, Signals and Devices 2020.

[3] Soltani, H.; Bel Hadj Ali, S.; and El Harabi, R. " $H_{\infty}$ Fault tolerant control for uncertain state time-delay systems", International Multi-conference on systems, Signals and Devices 2015, Sfax, Tunisia, pp. 1-6. 
[4] Abdelkrim, N.; Gassara, H.; and Chaabane, M. "Robust stabilization of time delay systems with polytopic-type uncertainties: lmi approach". The 4th International conference on systems and control 2015, 28-30 April. Sousse, Tunisia.

[5] Benjemaa, R.; Elhsoumi, A.; and Bel Hadj Ali, S. "Robust and PIM Control for Transmission Line with variable time delay". In the International conference on Sciences and Techniques of Automatic, control computer engineering (STA) 2020.

[6] Blanke, M.; Lunze, J.; Staroswiecki, M.; and Schroder, J. "Diagnosis and Fault-tolerant Control". Springer-Vcrlag, 2006, Berlin.

[7] Sun, X.; and Zhao, J. "Robust adaptive control for a class of nonlinear uncertain neutral delay systems", in Proceedings of American Control Conference 2004, Boston, Massachusetts, pp.609-613.

[8] Kaveh, M.; and Amir, G.A. “Adaptive robust control of uncertain neutral time-delay systems". American Control Conference 2008, Washington, USA, pp. 5162-5167.

[9] Carlos, E.; and Xi, L. "Delay-dependent robust $H_{\infty}$ control for uncertain linear state delayed systems", in Automatica 1999, vol. 35, No. 13, pp.1313-1321.

[10] Xu, S.; Shi, P.; Chu, Y.; and Zou, Y. "Robust stochastic stabilization and $H_{\infty}$ control of uncertain neutral stochastic time delay systems". Journal of Mathematical Analysis and Applications 2006, vol. 314, No. 1, pp. 1-16.

[11] Wei, Q.; Juan, L.; and Shumin, F. "Stability for uncertain neutral systems with time-varying delay". Chinese Control and Decision Conference 2010, Xuzhou, China, pp.1963-1965. 